\title{
Serum levels of inflammatory markers in type 2 diabetes patients with chronic periodontitis
}

\author{
Priscila Larcher LONGO ${ }^{1}$, Hilana Paula Carillo ARTESE ${ }^{2}$, Mariana de Sousa RABELO², Dione KAWAMOTO ${ }^{1}$, Adriana \\ Moura $\mathrm{FOZ}^{2}$, Giuseppe Alexandre ROMITO², Sérgio Atala DIB ${ }^{3}$, Marcia Pinto Alves MAYER ${ }^{1}$
}

1- Department of Microbiology, Institute of Biomedical Sciences, University of São Paulo, São Paulo, SP, Brazil.
2- Division of Periodontics, Department of Stomatology, School of Dentistry, University of São Paulo, São Paulo, SP, Brazil.
3- Department of Endocrinology, School of Medicine, Federal University of São Paulo, São Paulo, SP, Brazil.

Corresponding address: Priscila Larcher Longo - Departamento de Microbiologia, Instituto de Ciências Biomédicas - Universidade de São Paulo - Av. Lineu Prestes, 1374 - São Paulo - SP - 05508-900 - Brazil - Phone: 55-11-3091-7348 - e-mail: pllongo@usp.br

Submitted: September 28, 2013 - Modification: December 5, 2013 - Accepted: December 12, 2013

\section{ABSTRACT}

\begin{abstract}
$\mathrm{D}$ iabetes has been associated with periodontitis, but the mechanisms through which periodontal diseases affect the metabolic control remain unclear. Objective: This study aimed to evaluate serum levels of inflammatory markers, IL-8, IL-6 and monocyte chemoattractant protein 1 (MCP-1), in type 2 diabetic patients in the presence of chronic periodontitis. Material and Methods: Forty two individuals were enrolled in this study and assigned to one of five groups: diabetes mellitus with inadequate glycemic control and periodontitis (DMI+P, $n=10$ ), diabetes mellitus with adequate glycemic control and periodontitis ( $D M A+P, n=10)$, diabetes mellitus without periodontitis $(D M, n=10)$, periodontitis without diabetes $(P, n=6)$, and neither diabetes nor periodontitis $(H, n=6)$. Periodontal clinical examination included visible plaque index ( $P L)$, gingival bleeding index (GB), probing depth (PD), attachment level ( $A L$ ) and bleeding on probing (BP). Glycemic control was evaluated by serum concentration of glycated hemoglobin (HbA1c). Inflammatory serum markers IL-8, IL-6 and (MCP-1) were measured by ELISA. Results: $D M I+P$ and DMA+P groups presented higher PD $(p=0.025)$ and $A L(p=0.003)$ values when compared to the $\mathrm{P}$ group. There were no significant differences among groups for IL-6, IL-8 and MCP-1 serum levels. Conclusions: Although periodontitis was more severe in diabetic patients, the serum levels of the investigated inflammatory markers did not differ among the groups.
\end{abstract}

Keywords: Diabetes mellitus. Periodontitis. Cytokines. Inflammation.

\section{INTRODUCTION}

Type 2 diabetes mellitus (T2DM) is the most common endocrine disorder and its incidence is increasing worldwide. This condition is a serious public health concern due to the need for lifelong care, premature death and the fact that it remains incurable ${ }^{22}$. It is characterized by a progressive deficient secretion and action of insulin. Insulin resistance, whereby target tissues do not respond to this hormone, is a characteristic of the disease's first phase, when there is often a corresponding hyperinsulinemia ${ }^{1}$. This resistance comes together with inflammatory processes in the development of diabetes chronic complications ${ }^{32}$. Among these complications, periodontal diseases (PD) are very common and immune modulating factors are necessary for pathogen clearance, but also contribute to host tissues damage, as those seen in PD.

Clues to the involvement of inflammation in diabetes date back to more than a century ago. Proinflammatory molecules as tumor necroses factor (TNF)-a, leptin, interleukin (IL)-6, resistin, monocyte chemoattractant protein-1 (MCP-1) and visfatin, among others, are expressed at high levels in activated macrophages and/or other cells. Proinflammatory cytokines such as TNF-a and IL-1 $\beta$ activate JNK and IKK $\beta / N F-K B$ through classical receptor-mediated mechanisms which are also activated by pattern recognition receptors, bound to substances as lipopolissacharide (LPS) from gram negative bacteria. These include the Toll Like Receptors (TLRs) and the receptor for advanced glycation end products (RAGE). Prolonged 
hyperglycemia and the accompanying production of excess amounts of advanced glycation end products (AGEs) can activate NF-KB. JNK promotes insulin resistance through the phosphorylation of serine residues in insulin receptor signaling (IRS) -1 that negatively regulates normal signaling through the insulin receptor/IRS-1 axis. NF-KB induces insulin resistance by promoting the expression of numerous target genes as those for TNF- $\alpha$, IL-6, IL-8, MCP-1, MIP-1a, MIP-2, resistin, ICAM-1, VCAM-1 ${ }^{29}$.

The inflammation associated with $\mathrm{PD}$, characterized by elevated pro-inflammatory cytokines, innate immune receptor expression, and cellular infiltrate is exacerbated in patients with T2DM where TLR-4 and RAGEs play a significant role and contribute to induce responses by oral epithelial cells².

The local production of cytokines in response to periodontal bacteria and their products results in higher serum concentrations of pro-inflammatory biomarkers ${ }^{13}$ and a poor glycemic control in T2DM patients is associated with a loss of mucosal barrier integrity and accumulation of innate immune receptor ligands resulting in an exacerbation of ongoing inflammation ${ }^{2}$.

Thus, the adverse effect of periodontitis in T2DM may be explained by an increase in systemic inflammation, which contributes to insulin resistance ${ }^{19}$.

The interrelation between more severe PD and DM is established 8,32 and there are evidences on the importance of cytokine analysis in T2DM evolution ${ }^{29}$ and therapy blockade ${ }^{21}$.

As chronic or recurring inflammation contributes to an aberrant continuation of acute phase response and may also lead to further diabetes complications, such as micro and macroangiopathy and impaired healing ${ }^{14,28}$, it is suggested that periodontal disease with increased inflammatory response at local and systemic levels ${ }^{30}$ may collaborate to insulin resistance present on T2DM pathogenesis. However, scientific evidence on the effects of chronic periodontitis on diabetes mellitus remains inadequate and inconclusive ${ }^{9}$. Furthermore, whether periodontal therapy may help to control serum levels of inflammatory cytokines still remains controversial ${ }^{5}$. While some studies showed an effective improvement in periodontal parameters, circulating inflammatory markers and glycemic control after periodontal treatment in patients with $\mathrm{T}_{2} \mathrm{DM}^{9,17,32}$, others have shown that these responses were inconsistent across individuals and not sustainable over time ${ }^{3,5,19}$.

The elevated levels of systemic inflammatory mediators, such as IL-6, in obesity or metabolic syndrome enhance the host response to periodontal pathogens, hence increasing the chance of periodontal destruction. IL-6 is a multifunctional cytokine produced by a variety of cells including macrophages, neutrophils, and endothelial cells. The double edge effects (i.e., pro- and anti-inflammatory) of this molecule entail complexity in investigating its role and, until now, no evidence from animal or human studies supports the hypothetical model in which metabolic syndrome-induced IL- 6 increases the risk of destructive periodontal disease ${ }^{18}$.

Interleukin 8 (IL-8) is a chemokine important for recruiting neutrophils during healing and its levels were shown to be tightly linked to increased susceptibility to periodontitis ${ }^{12}$.

Monocyte chemoattractant protein 1 (MCP1 ) is also thought to play an important role in inflammatory processes. It has been implicated as a key factor in recruitment and activation of peripheral blood leukocytes in atherosclerotic lesions and adipose tissue. Elevated levels of circulating MCP-1 have been found in patients with T2DM, and experimental data have shown that this protein increases macrophage infiltration, inflammation and insulin resistance in transgenic mice ${ }^{26}$.

Some studies $3,6,9,17,24$ have evaluated markers such as interleukin-6 (IL-6), tumor necrosis factor-a (TNF- $\alpha$ ), C-reactive protein (CRP), and IL-1 $\beta$ and related them to insulin resistance ${ }^{24}$ and glycemic control $^{3}$; however, their results are still conflicting.

Based on the evidences that IL-6, IL- 8 and MCP1 are important key markers of immune response, which have been considered as critical mediators of initiation, progression and/or suppression of chronic periodontitis, and that no previous study had analyzed this combination of mediators in groups with and without diabetes and periodontal disease, this study aimed to evaluate serum levels of inflammatory markers, IL-8, IL- 6 and MCP-1, in type 2 diabetic patients in the presence of chronic periodontitis.

\section{PATIENTS AND METHODS}

This study was conducted between January 2012 and March 2013 in full accordance with the Helsinki Declaration of 1975, as revised in 2000. The study protocol and the informed consent form were reviewed and approved by the Biomedical Sciences Institute Ethics Committee (\#011/CEP) from the University of São Paulo (São Paulo, Brazil). T2DM individuals were recruited from the Diabetes Center at the Federal University of São Paulo. Non-diabetic patients were recruited from the School of Dentistry, University of São Paulo. Detailed medical and dental history was obtained from all patients. All volunteers received full mouth periodontal clinical examination performed at six sites per tooth (excluding third molars) from a calibrated examiner (HPCA). Intraexaminer reliability for detecting PDs within $1 \mathrm{~mm}$ was $>90 \%$. The presence of supragingival biofilm was recorded as plaque index $(P L)$, marginal gingival bleeding was recorded as gingival bleeding 
(GB); bleeding on probing (BP), probing depth (PD) and attachment level $(A L)$ were also evaluated. The probe used was North Caroline (UNC-15, Hu-Friedy ${ }^{\circledR}$, Chicago, IL, USA). PL, GB and BP were recorded as absent (0) or present (1). Inclusion criteria were: age $\geq 35$ years, confirmed diagnosis of type 2 diabetes over 3 years (for individuals with T2DM), generalized moderate to severe chronic periodontitis (American Academy of Periodontology, 1998) ( $\geq 30 \%$ of sites with $\mathrm{PD}>4 \mathrm{~mm}$ and bleeding on probing) and $\geq 15$ remaining teeth. Pregnant women, smokers, patients with Body Mass Index (BMI) $>40 \mathrm{~kg} / \mathrm{m}^{2}$ or who received periodontal therapy, antibiotic or antiseptic therapy 6 months prior to the study were excluded. Blood samples were obtained and glycated hemoglobin (HbA1c) levels were determined for all individuals at the clinical laboratory of the University of São Paulo Hospital. Approximately $5 \mathrm{ml}$ of blood were collected by venipuncture in untreated tubes (BD Vacutainer Rapid Serum Tube, Becton Dickinson Co., São Paulo, SP, Brazil). Serum was obtained by centrifugation at $2,500 \mathrm{~g}$ for 2 minutes, aliquoted and stored at $-80^{\circ} \mathrm{C}$ until further analysis. Quantitative measurement of IL-6, IL- 8 and MCP- 1 was assayed by enzyme-linked immunosorbent assay (Human Standard ELISA Development Kit, PeproTech Inc., Rocky Hill, CT, USA) and read out using a Micro Plate reader (Model 680, Bio-Rad Laboratories Inc., Hercules, CA, USA). Serum from each patient was tested in triplicate, and a calibration curve was added to each plate.

Forty-two individuals were enrolled in this study. Patients with T2DM and periodontitis were divided in two groups, as adequate glycemic control when $\mathrm{HbA} 1 \mathrm{c}<8.0 \%$ (DMA+P, $\mathrm{n}=10$ ) and inadequate glycemic control when $\mathrm{HbA} 1 \mathrm{c} \geq 8.0 \%$ (DMI+P, $n=10)$. The other 3 groups were composed of T2DM individuals without periodontitis $(D M, n=10)$, nondiabetic with periodontitis $(P, n=6)$ and non-diabetic without periodontitis $(H, n=6)$.

Periodontal clinical data were analyzed using appropriate statistical software (SPSS version 17.0 for Windows, SPSS Inc., Chicago, IL, USA) considering the individual as a study unit. The Kolmogorov-Smirnov test was used to test the variables normality distribution. Data were analyzed by non-parametric statistical methods since data were not normally distributed. Comparisons between groups were determined using Kruskal-Wallis test and Tukey test. Tests were based on median values with variability measures ( $25 \%$ and $75 \%$ quartiles). Results were considered statistically significant at $p<0.05$. For GB, PL and BOP data, percentages of positive sites were obtained per patient and, thereafter, median values were calculated for the groups. For $P D$ and $A L$, measured by millimeters, percent frequency was first obtained per patient and, thereafter, as a median value for the group.

Data from ELISA were analyzed by Kruskal-Wallis and Dunn's Multiple Comparison Test (Prism 5.0 Project, Graph Pad Software Inc., La Jolla, CA, USA). Values were obtained per patient and a median value was calculated for each group.

\section{RESULTS}

A total of 546 patients were assessed for eligibility, and only 42 fulfilled the inclusion criteria (Figure 1).

Demographic and $\mathrm{HbA} 1 \mathrm{c}$ data from each group are described in Table 1.

Table 2 provides periodontal clinical parameters

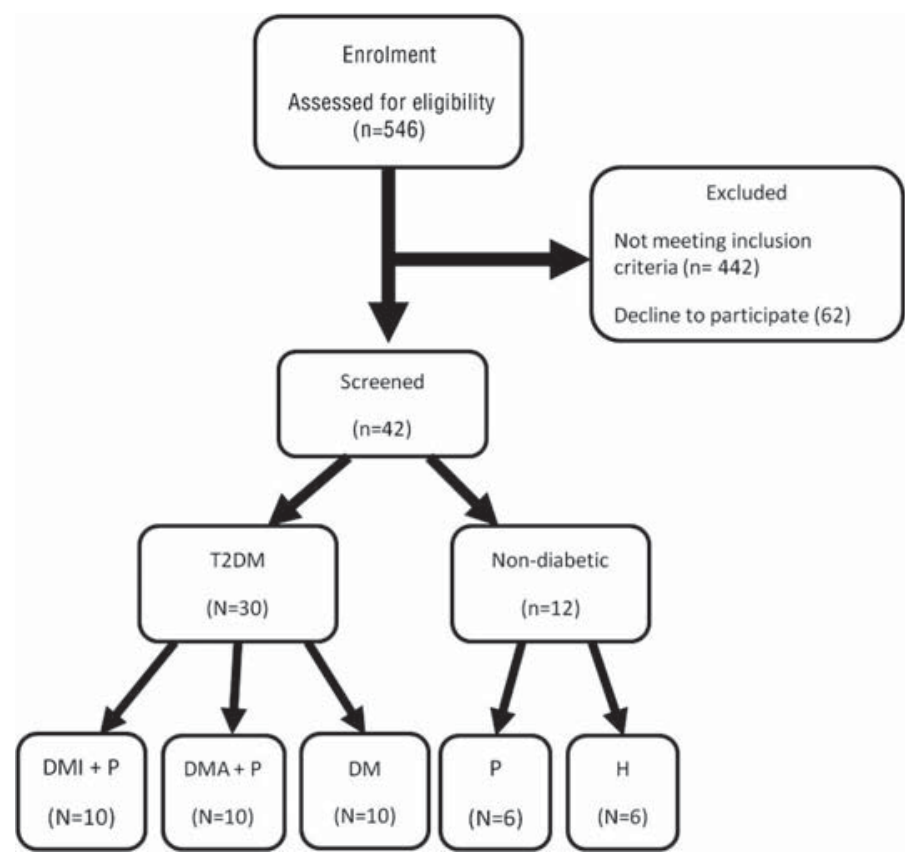

Figure 1- Flow diagram of patients included in the study 
for each group with periodontitis: $D M I+P, D M A+P$ and $P$. There were significant differences between diabetic (DMI+P and $D M A+P)$ and non-diabetic $(P)$ for $P D(p=0.04)$ and $A L(p=0.01)$ values indicating a more severe disease among the diabetic patients. The other clinical parameters did not differ significantly among groups. Intergroup clinical parameters comparison for $\mathrm{DMI}+\mathrm{P}$ and $\mathrm{DMA}+\mathrm{P}$ showed no significant differences (data not shown).

There were no differences between groups for IL-6 $(p=0.6351)$, IL-8 $(p=0.9460)$ and MCP-1 $(p=0.2987)$ sera levels (Figure 2$)$.

Table 1- Demographic characteristics and glycated level (mean \pm standard deviation) for participants in each group

\begin{tabular}{ccccc}
\hline GROUPS & male & age & BMI & HbA1c \\
\hline$H(n=6)$ & 2 & $50.0 \pm 4.38$ & $26.19 \pm 3.61$ & $5.18 \pm 0.60$ \\
$P(n=6)$ & 5 & $47.0 \pm 5.25$ & $26.18 \pm 3.28$ & $5.43 \pm 0.54$ \\
$D M(n=10)$ & 6 & $57.2 \pm 8.80$ & $26.18 \pm 2.37$ & $7.33 \pm 0.78$ \\
$D M A+P(n=10)$ & 6 & $60.6 \pm 10.67$ & $27.34 \pm 4.38$ & $6.83 \pm 0.78$ \\
$D M I+P(n=10)$ & 4 & $52.7 \pm 5.54$ & $28.92 \pm 6.36$ & $10.86 \pm 2.21$ \\
\hline
\end{tabular}

$\mathrm{DM}=$ type 2 diabetes mellitus; $\mathrm{P}=$ Periodontitis; $\mathrm{BMl}=$ body mass index. Groups: H (DM-P-), P (DM-P+), DM (DM+P-), $\mathrm{DMA}+\mathrm{P}(\mathrm{DM}+$ with adequate glycemic control $\mathrm{P}+), \mathrm{DMI}+\mathrm{P}(\mathrm{DM}+$ with inadequate glycemic control $\mathrm{P}+)$

Table 2- Medians and quartiles (25-75\%) of clinical parameters for the DMA+P, DMI+P and P groups

\begin{tabular}{ccccc}
\hline $\begin{array}{c}\text { Clinical } \\
\text { Parameters }\end{array}$ & $\begin{array}{c}\text { DMA+P } \\
(\mathbf{n}=10)\end{array}$ & $\begin{array}{c}\text { DMI+P } \\
(\mathbf{n}=10)\end{array}$ & $\begin{array}{c}\mathbf{P} \\
(\mathbf{n}=6)\end{array}$ & value \\
\hline GB $(\%)$ & $71.35(55.40-94.02)$ & $59.02(40.16-90.69)$ & $58.85(40.22-72.22)$ & 0.34 \\
PL (\%) & $90.83(68.00-97.90)$ & $66.70(52.52-97.00)$ & $66.88(52.40-81.62)$ & 0.25 \\
BOP (\%) & $77.56(68.24-94.54)$ & $67.55(40.52-93.68)$ & $58.80(43.87-65.82)$ & 0.88 \\
PD (mm) & $3.60(3.17-4.10)$ & $3.40(3.00-4.10)$ & $3.00(2.87-3.22)$ & $0.04^{*}$ \\
AL (mm) & $4.45(3.82-5.05)$ & $4.20(3.70-4.90)$ & $3.4(3.25-3.65)$ & $0.01^{*}$ \\
\hline
\end{tabular}

*Statistically significance difference between DMA+P;DMI+P and P (Kruskall-Wallis Test and Tukey Test; $\alpha=5 \%$ ). GB= gingival bleeding index; $\mathrm{PL}=$ visible plaque index; $\mathrm{BOP}=$ bleeding on probing; $\mathrm{PD}=$ probing depth; $\mathrm{AL}=$ attachment level

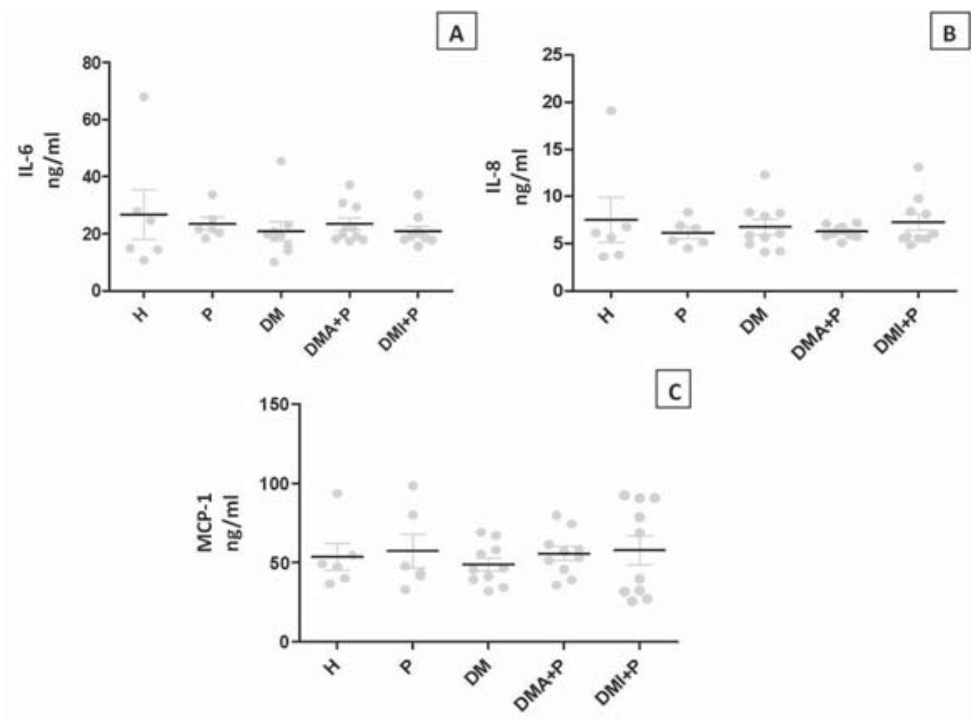

Figure 2- IL-6 (A), IL-8 (B) and MCP-1 (C) serum levels in patients without periodontitis and T2DM (H), patients with periodontitis and without T2DM (P), patients with T2DM and without periodontitis (DM), patients with T2DM with adequate glycemic control and periodontitis (DMA+P) and patients with T2DM with inadequate glycemic control and periodontitis (DMI+P). MCP-1=monocyte chemoattractant protein-1; IL-6=interleukin 6; IL-8=interleukin 8; T2DM=Type 2 diabetes mellitus. 


\section{DISCUSSION}

The present study evaluated clinical and inflammatory parameters among groups with and without T2DM and/or periodontitis.

T2DM patients presented significantly higher $P D$ and $A L$ values when compared to group $P$, without diabetes and with periodontitis. This result is consistent with a number of cross-sectional and longitudinal studies $16,20,31$ which had shown that the increased severity of PD in T2DM patients is due to an interorgan crosstalk under inflammatory conditions ${ }^{18}$.

The positive correlation between glycemic control and severity of periodontal disease has been reported in the literature ${ }^{8,15,34}$. Thus, we had expected to find a significant relationship between $\mathrm{HbA} 1 \mathrm{c}$ levels (representing adequate - $\mathrm{HbA} 1 \mathrm{c}<8.0 \%$ - and inadequate - HbA1c $\geq 8.0 \%$ - glycemic control) and periodontitis severity (in terms of increased PD and BOP). However, the periodontal clinical parameters data did not differ according to the diabetes control, corresponding to DMA+P and $\mathrm{DMI}+\mathrm{P}$ groups, respectively. We can speculate that, in order to observe correlation, we would need to consider a stricter glycemic control of individuals with T2DM, that is, HbA1c between 6.5 and $7.0 \%$, which would hamper even more the group selection. Besides, HbA1c refers to glycemic control in the last 2 or 3 months and we do not know how the chronic glycemic control in these individuals was. We also have to point out that hyperglycemia is only one factor of the multifactorial process of periodontal disease.

There were no differences among IL- 6 serum levels in the 5 groups analyzed. The role of locally produced IL- 6 in the periodontitis pathogenesis has been demonstrated ${ }^{4,25}$, since high levels of this cytokine were detected in symptomatic large lesions rather than in asymptomatic small lesions ${ }^{4}$. This cytokine is overexpressed in sites with PD mainly in patients without diabetes and patients with wellcontrolled T2DM when compared with patients with poorly controlled diabetes ${ }^{35}$. The effect of periodontal treatment on IL-6 levels showed conflicting data, yielding a tendency toward a decrease ${ }^{7,32}$ or increase in serum levels in T2DM patients ${ }^{33}$. Furthermore, a controversial study suggested an increase or no change in serum IL-6 levels in obesity and metabolic syndrome ${ }^{18}$. It should be noticed that T2DM individuals evaluated in the present study were under treatment with insulin, which may have induced low levels of inflammatory biomarkers such as IL-6 6,23 , counteracting the high levels induced by periodontal inflammation.

In the present study, there were no significant differences in the sera levels of the studied inflammatory markers, MCP-1 and IL-8, among the groups, although previous data demonstrated that both were overexpressed in the gingival tissue with chronic periodontitis ${ }^{35}$. A search in the literature revealed no study in humans regarding T2DM and/ or periodontitis and MCP-1 serum or gingival fluid levels. In rats experimental models, MCP-1 gingival crevicular fluid concentrations did not significantly differ between groups with diabetes and periodontitis and its levels were higher than in control healthy group ${ }^{27}$.

High levels of IL-8 were shown in the gingival fluid of subjects with periodontal disease. However, subjects with T2DM presented significantly lower levels of IL-8 in the GCF when compared to healthy ones and there was no correlation between IL-8 gingival levels and $\mathrm{HbA} 1 \mathrm{c}$ level ${ }^{10,11}$. Furthermore, IL-8 levels in serum tended to decrease after periodontal therapy in T2DM patients ${ }^{7}$.

Due to the use of restrictive inclusion and exclusion criteria, in an attempt to minimize the occurrence of confounding factors, the small sample size is one limitation of this study. However, it is interesting to consider that some studies evaluated only specific dental sites through gingival crevicular fluid analysis, whereas the serum analysis provides a more valuable analysis of the inflammatory markers in the subject. To the best of our knowledge, there have been no studies evaluating these serum markers under all different conditions analyzed in this study.

Nevertheless, this study confirms the higher severity of periodontal disease in individuals with diabetes, highlighting the need for special oral healthcare for these patients. This study pointed out the great variability of the serum inflammatory biomarkers under all systemic and periodontal conditions studied. We can suggest that follow-up studies with stricter glycemic control and larger samples should be conducted in order to investigate the cumulative influence of periodontal conditions on serum inflammatory biomarkers in individuals with T2DM.

\section{CONCLUSION}

Although periodontitis was more severe in patients with diabetes, the serum levels of the investigated inflammatory markers were not different among the periodontitis patients.

\section{ACKNOWLEDGMENTS}

We would like to thank Dr. Anna Carolina R. T. Horliana and Dr. Giovane H. Gomes for their collaboration in clinical procedures. This study was supported by São Paulo Research Foundation (FAPESP), São Paulo, Brazil, under protocol numbers 2011/18618-5; 10057-4; 06982-4. 


\section{REFERENCES}

1- American Diabetes Association. Standards of medical care in diabetes - 2011. Diabetes Care. 2011;34(Suppl 1):S11-61.

2- Amir J, Waite M, Tobler J, Catalfamo DL, Koutouzis T, Katz J, et al. The role of hyperglycemia in mechanisms of exacerbated inflammatory responses within the oral cavity. Cell Immunol. 2011;272(1):45-52.

3- Auyeung L, Wang PW, Lin RT, Hsieh CJ, Lee PY, Zhuang RY, et al. Evaluation of periodontal status and effectiveness of non-surgical treatment in patients with type 2 diabetes mellitus in Taiwan for a 1-year period. J Periodontol. 2012;83(5):621-8.

4- Azuma MM, Samuel RO, Gomes-Filho JE, Dezan-Junior E, Cintra LT. The role of IL- 6 on apical periodontitis: a systematic review. Int Endod J. 2013. doi: 10.1111/iej.12196. Epub ahead of print. 5- Behle JH, Sedaghatfar MH, Demmer RT, Wolf DL, Celenti R, Kebschull $M$, et al. Heterogeneity of systemic inflammatory responses to periodontal therapy. J Clin Periodontol. 2009;36(4):287-94.

6- Beisswenger PJ, Brown WV, Ceriello A, Le NA, Goldberg RB, Cooke JP, et al. Meal induced increases in C-reactive protein, interleukin- 6 and tumour necrosis factor a are attenuated by prandial + basal insulin in patients with type 2 diabetes. Diabet Med. $2011 ; 28(9): 1088-95$.

7- Correa FO, Gonçalves D, Figueredo CM, Bastos AS, Gustafsson A, Orrico SR. Effect of periodontal treatment on metabolic control, systemic inflammation and cytokines in patients with type 2 diabetes. J Clin Periodontol. 2010;37(1):53-8.

8- Costa FO, Miranda Cota LO, Pereira Lages EJ, Soares Dutra Oliveira AM, Dutra Oliveira PA, Cyrino RM, et al. Progression of periodontitis and tooth loss associated with glycemic control in individuals undergoing periodontal maintenance therapy: a 5-year follow-up study. J Periodontol. 2013;84(5):595-605.

9- Chen L, Luo G, Xuan D, Wei B, Liu F, Li J, et al. Effects of non-surgical periodontal treatment on clinical response, serum inflammatory parameters, and metabolic control in patients with type 2 diabetes: a randomized study. J Periodontal. 2012;83(4):435-43.

10- Duarte PM, Oliveira MC, Tambeli CH, Parada CA, Casati MZ, Nociti FH Jr. Overexpression of interleukin-1beta and interleukin-6 may play an important role in periodontal breakdown in type 2 diabetic patients. J Periodontal Res. 2007;42(4):377-81.

11- Engebretson SP, Vossughi F, Hey-Hadavi J, Emingil G, Grbic JT. The influence of diabetes on gingival crevicular fluid beta-glucuronidase and interleukin-8. J Clin Periodontol. 2006;33(11):784-90.

12- Figueredo CM, Gustafsson A. Increased amounts of laminin in GCF from untreated patients with periodontitis. J Clin Periodontol. $2000 ; 27(5): 313-8$.

13- Górska R, Gregorek H, Kowalski J, Laskus-Perendyk A, Syczewska M, Madaliński K. Relationship between clinical parameters and cytokine profiles in inflamed gingival tissue and serum samples from patients with chronic periodontitis. J Clin Periodontol. 2003;30(12):1046-52.

14- Graves DT, Kayal RA. Diabetic complications and dysregulated innate immunity. Front Biosci. 2008;13(1):1227-39.

15- Haseeb M, Khawaja KI, Ataullah K, Munir MB, Fatima A. Periodontal disease in type 2 diabetes mellitus. J Coll Physicians Surg Pak. 2012;22(8):514-8.

16- Heitz-Mayfield LJ. Disease progression: identification of highrisk groups and individuals for periodontitis. J Clin Periodontol. 2005;32(Suppl 6):196-209.

17- Kardeşler L, Buduneli N, Cetinkalp S, Kinane DF. Adipokines and inflammatory mediators after initial periodontal treatment in patients with type 2 diabetes and chronic periodontitis. J Periodontol. 2010;81(1):24-33.
18- Khosravi R, Ka K, Huang T, Khalili S, Nguyen BH, Nicolau B, et al. Tumor necrosis factor- $a$ and interleukin-6: potential interorgan inflammatory mediators contributing to destructive periodontal disease in obesity or metabolic syndrome. Mediators Inflamm. 2013;2013:728987.

19- Koromantzos PA, Makrilakis K, Dereka X, Offenbacher S, Katsilambros N, Vrotsos IA, et al. Effect of non-surgical periodontal therapy on C-reactive protein, oxidative stress, and matrix metalloproteinase (MMP)-9 and MMP-2 levels in patients with type 2 diabetes: a randomized controlled study. J Periodontol. 2012;83(1):3-10.

20- Lalla E, Papapanou PN. Diabetes mellitus and periodontitis: a tale of two common interrelated diseases. Nat Rev Endocrinol. $2011 ; 7(12): 738-48$.

21- Larsen CM, Faulenbach M, Vaag A, Ehses JA, Donath MY, Mandrup-Poulsen T. Sustained effects of interleukin-1 receptor antagonist treatment in type 2 diabetes. Diabetes Care. 2009;32(9):1663-8.

22- Litwak L, Goh SY, Hussein Z, Malek R, Prusty V, Khamseh ME. Prevalence of diabetes complications in people with type 2 diabetes mellitus and its association with baseline characteristics in the multinational A1chieve study. Diabetol Metab Syndr. 2013;5(1):57. 23- Mao XM, Liu H, Tao XJ, Yin GP, Li Q, Wang SK. Independent antiinflammatory effect of insulin in newly diagnosed type 2 diabetes. Diabetes Metab Res Rev. 2009;25(5):435-41.

24- Mealey B, Oates T; American Academy of Periodontology. Diabetes mellitus and periodontal diseases. J Periodontol. $2006 ; 77(8): 1289-303$

25- Nibali L, Fedele S, D'Aiuto F, Donos N. Interleukin-6 in oral diseases: a review. Oral Dis. 2012;18(3):236-43.

26- Odegaard JI, Ricardo-Gonzalez RR, Goforth MH, Morel CR, Subramanian V, Mukundan L, et al. Macrophage-specific PPARgamma controls alternative activation and improves insulin resistance. Nature. 2007;447(7148):1116-20.

27- Sakallioğlu EE, Ayas B, Lütfioğlu M, Keleş GC, Açikgöz G, Firatli E. Gingival levels of monocyte chemoattractant protein-1 (MCP-1) in diabetes mellitus and periodontitis: an experimental study in rats. Clin Oral Investig. 2008;12(1):83-9.

28- Saremi, A, Nelson RG, Tulloch-Reid M, Hanson RL, Sievers $M L$, Taylor GW, et al. Periodontal disease and mortality in type 2 diabetes. Diabetes Care. 2005;28(1):27-32.

29- Shoelson SE, Lee J, Goldfine AB. Inflammation and insulin resistance. J Clin Invest. 2006;116(7):1793-801.

30- Southerland JH, Moss K, Taylor GW, Beck JD, Pankow J, Gangula $P R$, et al. Periodontitis and diabetes associations with measures of atherosclerosis and CHD. Atherosclerosis. 2012;222(1):196-201. 31- Southerland JH, Taylor GW, Moss K, Beck JD, Offenbacher S. Commonality in chronic inflammatory diseases: periodontitis, diabetes, and coronary artery disease. Periodontol 2000. 2006;40:130-43.

32- Sun WL, Chen LL, Zhang SZ, Wu YM, Ren YZ, Qin GM. Inflammatory cytokines, adiponectin, insulin resistance and metabolic control after periodontal intervention in patients with type 2 diabetes and chronic periodontitis. Intern Med. 2011;50(15):1569-74.

33- Talbert J, Elter J, Jared HL, Offenbacher S, Southerland J, Wilder RS. The effect of periodontal therapy on TNF-alpha, IL-6 and metabolic control in type 2 diabetics. J Dent Hyg. 2006;80(2):7-22. 34- Tanwir F, Tariq A. Effect of glycemic control on periodontal status. J Coll Physicians Surg Pak. 2012;22(6):371-4.

35- Venza I, Visalli M, Cucinotta M, De Grazia G, Teti D, Venza M. Proinflammatory gene expression at chronic periodontitis and peri-implantitis sites in patients with or without type 2 diabetes. J Periodontol. 2010;81(1):99-108. 